\title{
APPLICATIONS OF SARS-COV-2 SEQUENCING DATA
}

\author{
Cláudia Oliveira da Silva ${ }^{1}$, Tatiana Donai Lopes ${ }^{1}$ and Brígida Mónica Faria ${ }^{1,2}$ \\ ${ }^{I}$ Escola Superior de Saúde - Politécnico do Porto (ESS-P.Porto), Portugal \\ ${ }^{2}$ Laboratório de Inteligência Artificial e Ciência de Computadores (LIACC), Portugal
}

\begin{abstract}
Monitoring and understanding pathogen epidemiology and evolution is crucial, especially during an outbreak. COVID-19 pandemic, caused by the newly identified virus SARS-CoV-2, is one of the current major challenges that require the best scientific and technological means. Secondary to sequencing, one of the most basic steps in the study of the many virus strains is sequence alignment. In this work, the focus was on the search and selection of a Biopython algorithm of global pairwise sequence alignment, its implementation and analysis of effectiveness and complexity. To apply this algorithm, which provides the level of similarity between sequences, it was used SARS-CoV-2 genomic sequences of samples collected in 10 countries to analyse its similarity with the reference sequence (NC_045512). There were obtained very high similarities between all sequences and it was observed a decrease of similarity over time in most of the countries included in this study. These results are concordant with recent ones that are publicly shared. In this context, the algorithm performed effectively and its low complexity will not limit its application in a bigger volume of data.
\end{abstract}

\section{KEYWORDS}

SARS-CoV-2, Pairwise Alignment, Similarity, Biopython

\section{INTRODUCTION}

Declared as a global public health emergency, on $11^{\text {th }}$ March 2020, by the World Health Organization, COVID-19 pandemic is one of the major human challenges of current time. This viral respiratory illness is caused by a new Coronavirus called SARS-CoV-2 (Phan 2020). Previously unknown, this is a Betacoronavirus discovered using sequencing in samples from patients with pneumonia. Phylogenetically, this novel virus forms a clade within the subgenus Sarbecovirus, Orthocoronavirinae subfamily and it is the seventh member of the family of coronaviruses that infect humans (Zhu, Zhang et al. 2020).

So far, epidemiological investigations have suggested that the outbreak is related to a seafood market in Wuhan, China (Wu, Zhao et al. 2020). The first virus genomes and associated data were publicly shared via GISAID $^{\odot}$ (https://www.gisaid.org/) on $10^{\text {th }}$ January 2020. It is crucial to monitor and understand pathogen epidemiology and evolution, specially during an outbreak. Rapid methods that can aid in the analysis, interpretation and dissemination of results are fundamental as sequencing is becoming faster and its costs are continually dropping (Hadfield, Megill et al. 2018). Sequence alignment is essential to study protein structures, to establish phylogenetic relations and inference of molecular evolution. The computational tools are extremely necessary to achieve accurate and faster results. Pairwise sequence alignment is one of the fundamental means in bioinformatics to assess the degree of similarity as well as to find differences, that may indicate functional, structural or evolutionary relations between two biological sequences (Muhire, Varsani et al. 2014). This tool forms the basis of many database search programs, such as BLAST.

In what concerns global sequence alignments, solutions have been proposed in the past to find the optimal global alignment between two sequences (that comprehends the total length of the sequences) by applying the concept of dynamic programming - for global alignment, the original algorithm is defined as Needleman-Wunsch by the names of the authors who first proposed it: Needleman and Wunsch, 1970 (Baichoo and Ouzounis 2017). Many bioinformatic tools use Python language. Python is a high-level programming language and available in open source. The Biopython project is a mature international collaboration of volunteer developers, providing Python libraries for a wide range of bioinformatic problems. Biopython includes a set of tools written in Python, which are used for read and writing different sequence file formats and multiple sequence alignments, among other features. It is freely available for all the major operating systems with documentation and source code (https://biopython.org) under the Biopython license (Cock, Antao et al. 2009). 
Concerning the similarity data, recent studies show equivalent results for the similarity between SARS-CoV-2 sequences. Wang et al obtained a similarity of 99,99\% (99,91\%-100\%) at the nucleotide level, among viral strains from different countries (China, America, Australia, Thailand, The United Kingdom, Germany, France, Finland, Korea, Singapore and others). These sequences were retrieved until $14^{\text {th }}$ February (Wang, Liu et al. 2020). Li et al studied the similarities between 194 COVID-19 strains from 21 countries had obtained an average similarity between any comparison of two sequences of $0.995561 / 0.995342$. It was also determined the average percentage similarities between sequences of the viral strains across samples collected from different months, and although the overall similarity of the human strains was high, it was observed a reduction of similarity in later months, indicating that mutations within the human population are occurring. In December 2019, the similarity was on average 0.999054 , corresponding to an average of 29,75 different nucleotides. In March 2020, this similarity had dropped to 0,988468, corresponding to an average of 348,33 different nucleotides. These differences show evolutional changes of the virus, which might imply attenuated or even more virulent strains (Li, Liu et al. 2020). The work of Randhawa et al also achieved over 99\% sequence similarity within the virus sequences, validating their common lineage and source (Randhawa, Soltysiak et al. 2020).

This work consisted of the search and selection of a Biopython algorithm, its implementation and analysis of its effectiveness and complexity. It was chosen a pairwise sequence alignment algorithm which performs a global alignment. Applying the algorithm, forward described, provides the level of similarity between sequences, were used SARS-CoV-2 genomic sequences of samples collected in 10 countries to analyse its similarity with the reference sequence. The main goal of this study was to apply an alignment bioinformatic tool to check its effectiveness by comparing its results with the ones that are being achieved and publicly shared.

\section{METHODS}

\subsection{Data}

To obtain the sequences for alignment, DNA-seq data of SARS-CoV-2 was collected from GISAID (the sequences were downloaded in FASTA format). There were used 20 complete genome sequences from SARS-CoV-2 virus, 2 per each country. The sequences were chosen based on collection data, the first sequence representing the earliest sequencing of SARS-CoV-2 in each country, and the second constituting the last sequencing published at the time of the study ( $8^{\text {th }}$ of June). The reference sequence (being a comparation method that will be explained later in the paper) was taken from NCBI (https://ncbi.nlm.nih.gov) (Table 1). Sequences that presented stretches of NNNs were excluded. There were included 10 countries in 6 continents: China, Thailand, India, Russia, Italy, Portugal, South Africa, United States of America, Brazil and Australia, represented by white squares in Figure 1. The choice of these countries is related to two inclusion criteria: having representation from all over the world, including at least one country for each continent, and the number of cases in these countries relatively high.

Table 1. Summary of the countries studied with Accession ID from GISAID and Collection Date

\begin{tabular}{|c|c|c|c|c|c|}
\hline Country & Accession ID & Collection date & Country & Accession ID & $\begin{array}{l}\text { Collection } \\
\text { date }\end{array}$ \\
\hline \multirow[t]{2}{*}{ Portugal } & $1^{\text {st }}$ EPI_ISL_413647 & $01 / 03 / 2020$ & \multirow[t]{2}{*}{ EUA } & $1^{\text {st-EPI_ISL_404895 }}$ & $19 / 01 / 2020$ \\
\hline & $2^{\text {nd }}-E P I$ ISL_454272 & $02 / 05 / 2020$ & & $2^{\text {nd }}-E P I+I S L=461476$ & $30 / 05 / 2020$ \\
\hline \multirow[t]{2}{*}{ China } & $1^{\text {st-EPI_ISL_402119 }}$ & 20/12/2019 & \multirow[t]{2}{*}{ India } & $1^{\text {st-EPI_ISL_413522 }}$ & $27 / 01 / 2020$ \\
\hline & $2^{\text {nd }}-E P I$ ISL_444969 & $16 / 04 / 2020$ & & $2^{\text {nd }}-E P I$ ISL_461506 & $27 / 05 / 2020$ \\
\hline \multirow[t]{2}{*}{ South Africa } & $1^{\text {st-EPI_ISL_417186 }}$ & 07/03/2020 & \multirow[t]{2}{*}{ Italy } & $1^{\text {st-EPI_ISL_412974 }}$ & $29 / 01 / 2020$ \\
\hline & $2^{\text {nd-EPI_ISL_455635 }}$ & 05/05/2020 & & $2^{\text {nd}}-E P I$ ISL_436732 & $27 / 04 / 2020$ \\
\hline \multirow[t]{2}{*}{ Brazil } & $1^{\text {st-EPI_ISL_413016 }}$ & 28/02/2020 & \multirow[t]{2}{*}{ Russia } & $1^{\text {st-EPI_ISL_460096 }}$ & $02 / 03 / 2020$ \\
\hline & $2^{\text {nd}}-E P I$ ISL_458149 & 06/04/2020 & & $2^{\text {nd }}-E P I$ ISL_450291 & $23 / 04 / 2020$ \\
\hline \multirow[t]{2}{*}{ Australia } & $1^{\text {st-EPI_ISL_407893 }}$ & $24 / 01 / 2020$ & \multirow[t]{2}{*}{ Thailand } & $1^{\text {st }}$-EPI_ISL_403962 & 08/01/2020 \\
\hline & $2^{\text {nd }}-E P I+I S L+456649$ & $27 / 05 / 2020$ & & $2^{\text {nd }}-E P I-I S L+447026$ & 03/04/2020 \\
\hline Reference & NC_045512 & $30 / 03 / 2020$ & & & \\
\hline
\end{tabular}




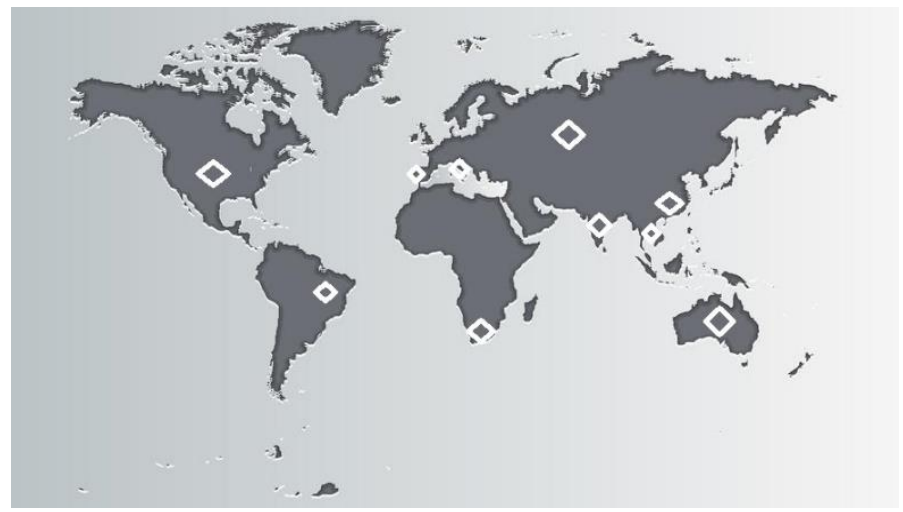

Figure 1. Representation of the geographic location of the countries of origin of the studied sequenced samples

The studied sequences were collected from patients diagnosed with COVID-19. Samples were taken from 20 patients, 8 women, 7 man and 5 unknowns, with a mean age of $48,6 \pm 21,74$.

\subsection{Sequence Alignment Algorithm}

To align and compare the sequences of this study, an algorithm in Python was adapted from Nagesh Singh Chauhan (https://github.com/nageshsinghc4/COVID-19-coronavirus). The code was assembled based on the module defined as pairwise 2. This module provides functions to get global and local alignments between two sequences, with the main goal of knowing the percentage of similarity between two sequences. The Bio.SeqIO module provides a simple interface for read and writing biological sequence files in various formats. Regardless of the file format, the information is held as SeqRecord objects (Cock, Antao et al. 2009).

In this study, the CPU time was measured in different points in the source code, with the aim of knowing how much CPU time was used to perform instructions. The computational equipment used was HP Pavilion Laptop 15- cw0xx. The implemented code is partially displayed in Figure 2, where it was named the pairwise2 instruction as "alghorithmSARS_COV'. The "first.seq" represents the first sequencing from each country, and "last.seq" the last sequencing.

alghoritmSARS_COV = pairwise2.align.globalxx(first.seq, last.seq, one_alignment_only=True, score_only=True)

print('SARS/COV Similarity (\%):', alghoritmSARS_COV / len(ref.seq) * 100)

Figure 2. Fragment of the implemented algorithm of the pairwise 2 module

This work was divided in two parts. In the first part, the last and first sequencing were compared to find the percentage of similarity between them; in the second part, the reference sequence was compared with the first and last sequencing from each country.

\section{RESULTS AND DISCUSSION}

In the first part of this work, the algorithm was executed for the two sequences from each country (first/last). The output of the code gave information about the percentage of similarity between the tested sequences. For all 10 countries the percentage of similarity was above $99 \%$, as presented on Table 2. 
Table 2. Summary data of the length and $\%$ of similarity of the first and last sequencing from each country

\begin{tabular}{|c|c|c|c|c|c|}
\hline Country & $\begin{array}{l}\text { Sequence length } \\
\text { (nucleotides) }\end{array}$ & $\%$ of similarity & Country & $\begin{array}{l}\text { Sequence length } \\
\text { (nucleotides) }\end{array}$ & $\%$ of similarity \\
\hline Portugal & $\begin{array}{l}1^{\text {st }}-29903 \\
2^{\text {nd }}-29763\end{array}$ & $99,49 \%$ & EUA & $\begin{array}{l}1^{\text {st }}-29882 \\
2^{\text {nd }}-29891\end{array}$ & $99,83 \%$ \\
\hline China & $\begin{array}{l}1^{\text {st }}-29891 \\
2^{\text {nd }}-29882\end{array}$ & $99,95 \%$ & India & $\begin{array}{l}1^{\text {st }}-29854 \\
2^{\text {nd }}-29903\end{array}$ & $99,87 \%$ \\
\hline South Africa & $\begin{array}{l}1^{\text {st }}-29903 \\
2^{\text {nd }}-29903\end{array}$ & $99,55 \%$ & Italy & $\begin{array}{l}1^{\text {st }}-29903 \\
2^{\text {nd }}-29874\end{array}$ & $99,78 \%$ \\
\hline Brazil & $\begin{array}{l}1^{\text {st }}-29890 \\
2^{\text {nd }}-29903\end{array}$ & $99,53 \%$ & Russia & $\begin{array}{l}1^{\text {st }}-29543 \\
2^{\text {nd }}-29903\end{array}$ & $99,97 \%$ \\
\hline Australia & $\begin{array}{l}1^{\text {st }}-29782 \\
2^{\text {nd }}-29903 \\
\end{array}$ & $99,31 \%$ & Thailand & $\begin{array}{l}1^{\text {st }}-29848 \\
2^{\text {nd }}-29782 \\
\end{array}$ & $99,01 \%$ \\
\hline
\end{tabular}

In the second part of the task, the compared sequences were the reference sequence from SARS-CoV-2 and the first (ref/first) and last (ref/last) sequence from each country. As seen on Table 3, all the similarity percentages presented values above $98 \%$. The average percentage of similarity between the reference sequence and first sequencing from each country was $99,89 \% \pm 0,0016$. The average percentage of similarity between the reference sequence and last sequencing from each country was $99,59 \% \pm 0,0039$.

Table 3. Summary data of the $\%$ of similarity of the first/last sequencing from each country with the reference sequence

\begin{tabular}{ccc}
\hline Country & $\begin{array}{c}\text { \% of similarity } \\
\text { (ref/first) }\end{array}$ & $\begin{array}{c}\text { \% of similarity } \\
\text { (ref/last) }\end{array}$ \\
\hline Portugal & $99,91 \%$ & $99,49 \%$ \\
China & $100,00 \%$ & $99,91 \%$ \\
South Africa & $99,90 \%$ & $99,49 \%$ \\
Brazil & $99,58 \%$ & $99,95 \%$ \\
Australia & $99,58 \%$ & $98,98 \%$ \\
EUA & $99,99 \%$ & $99,84 \%$ \\
India & $99,98 \%$ & $99,74 \%$ \\
Italy & $99,99 \%$ & $99,79 \%$ \\
Russia & $99,97 \%$ & $99,90 \%$ \\
Thailand & $100,00 \%$ & $98,83 \%$ \\
\hline
\end{tabular}

Statistical differences were calculated for the means displayed above. According to Shapiro-Wilk test, the normality was assumed $(p>0,05)$. The $t$-student test for paired samples was performed. The differences between the mean of ref/first and ref/last were statistically significative $(\mathrm{p}=0,044<0,05)$, for the differences between the mean of first/last and ref/last, were not statistically significative $(\mathrm{p}=0,557>0,05)$, what can be justified by the fact that the first sequences from some countries were coincident or close in time to the reference sequence. Finally, the differences between the mean of ref/first and first/last, were statistically significative $(\mathrm{p}=0,019<0,05)$. Except for Brazil and Australia results, in general, the similarities decreased over time, between the first and last sequences analysed in this work. This decrease may imply that are occurring evolutional changes of the virus, as supported by recent publicly shared results (Li, Liu et al. 2020, Randhawa, Soltysiak et al. 2020, Wang, Liu et al. 2020). These results were expected, since all the similarities values are analogous to those that are being achieved by the previously referenced works, therefore the algorithm applied in this study was considered effective. For this paper, the CPU time was measured for the function of the alignment (pairwise2.align.globalxx). Ten trials were made in order to get an average (Table 4). The average CPU time for the mentioned function was $23,76 \pm 0,72$.

Table 4. CPU times of each try

\begin{tabular}{cccc}
\hline Trial & CPU time & Trial & CPU time \\
\hline 1 & 23,95 & 6 & 22,92 \\
2 & 24,18 & 7 & 23,29 \\
3 & 24,90 & 8 & 24,79 \\
4 & 23,63 & 9 & 23,71 \\
5 & 22,66 & 10 & 23,62 \\
\hline
\end{tabular}


The algorithm performed with high efficiency, so its application in bigger volume of data will not be compromised.

\section{CONCLUSION}

In conclusion, with the time passing, the percentage of similarities between the studied sequences have been decreasing, however this was not verified in all the comparisons and countries. It can also be apprehended that SARS-CoV-2 is a virus with a relatively low mutation rate, being a great advantage for a therapeutic solution. Summing up, the pairwise alignment algorithm that was used in this project was effective and showed high efficiency, which indicates advantages in its application. Regardless of these features, in a pandemic context, the availability of even faster methodology, as machine learning methods can be very useful.

In future work, it would be valuable to extend the application of the algorithm to further collection dates and to other countries data, to verify if the sequence similarities would maintain equivalent to other published results. To implement and analyse another algorithm with this data, would also be interesting for comparison and eventually give some inputs to optimization and integration in programs that are already being applied.

\section{ACKNOWLEDGEMENT}

Authors would like to acknowledge Escola Superior de Saúde - P.Porto and LIACC. This research was partially supported by LIACC (FCT/UID/CEC/0027/2020).

\section{REFERENCES}

Baichoo, S. and C. A. Ouzounis (2017). Computational complexity of algorithms for sequence comparison, short-read assembly and genome alignment. Biosystems 156-157: 72-85.

Cock, P. J. A., T. Antao, J. T. Chang, B. A. Chapman, C. J. Cox, A. Dalke, I. Friedberg, T. Hamelryck, F. Kauff, B. Wilczynski and M. J. L. de Hoon (2009). Biopython: freely available Python tools for computational molecular biology and bioinformatics. Bioinformatics 25(11): 1422-1423.

Hadfield, J., C. Megill, S. M. Bell, J. Huddleston, B. Potter, C. Callender, P. Sagulenko, T. Bedford and R. A. Neher (2018). Nextstrain: real-time tracking of pathogen evolution. Bioinformatics 34(23): 4121-4123.

Li, Y., B. Liu, J. Cui, Z. Wang, Y. Shen, Y. Xu and K. Yao (2020). Similarities and Evolutionary Relationships of COVID-19 and Related Viruses. Available at: https://arxiv.org/abs/2003.05580

Muhire, B. M., A. Varsani and D. P. Martin (2014). SDT: a virus classification tool based on pairwise sequence alignment and identity calculation. PloS one 9(9): e108277-e108277.

Phan, T. (2020). Genetic diversity and evolution of SARS-CoV-2. Infection, genetics and evolution : journal of molecular epidemiology and evolutionary genetics in infectious diseases 81: 104260-104260.

Randhawa, G. S., M. P. M. Soltysiak, H. El Roz, C. P. E. de Souza, K. A. Hill and L. Kari (2020). Machine learning using intrinsic genomic signatures for rapid classification of novel pathogens: COVID-19 case study. PLoS One 15(4).

Wang, C., Z. Liu, Z. Chen, X. Huang, M. Xu, T. He and Z. Zhang (2020). The establishment of reference sequence for SARS-CoV-2 and variation analysis. J Med Virol.

Wu, F., S. Zhao, B. Yu, Y. Chen, W. Wang, Z.-G. Song, Y. Hu, Z.-W. Tao, J.-H. Tian, Y.-Y. Pei, M.-L. Yuan, Y.-L. Zhang, F.-H. Dai, Y. Liu, Q.-M. Wang, J.-J. Zheng, L. Xu, E. Holmes and Y.-Z. Zhang (2020). A new coronavirus associated with human respiratory disease in China. Nature 579: 1-8.

Zhu, N., D. Zhang, W. Wang, X. Li, B. Yang, J. Song, X. Zhao, B. Huang, W. Shi, R. Lu, P. Niu, F. Zhan, X. Ma, D. Wang, W. Xu, G. Wu, G. F. Gao and W. Tan (2020). A Novel Coronavirus from Patients with Pneumonia in China, 2019. N Engl J Med 382(8): 727-733. 